\title{
Generación de bienestar social en México: un estudio DEA a partir del IDH
}

\section{Generation of social welfare in Mexico: A DEA study from $\mathrm{HDI}$}

\author{
José CÉsar Navarro-Chávez* \\ Francisco JaVier Ayvar-Campos** \\ Víctor Manuel Giménez-García***
}

\begin{abstract}
The following document approaches the study of the efficient use of resources to generate social welfare in Mexico, during the period 1990-2010. For this it was used the Data Envelopment Analysis (DEA), taking as a reference the socio-economic indicators of the three dimensions of human development index (HDI). The analysis of results showed that Baja California Sur, Campeche, Colima, Chiapas, Mexico City, State of Mexico, Quintana Roo, Tlaxcala and Zacatecas were more efficient in generating social welfare. Becoming evident that entities which receive more resources are not necessarily those that produce higher social welfare.
\end{abstract}

Keywords: welfare, HDI, DEA, States of Mexico.

\section{Resumen}

El presente documento aborda el estudio del uso eficiente de los recursos para generar bienestar social en México durante el periodo 1990-2010; para ello se utilizó el Análisis de la Envolvente de Datos (DEA), tomando como referencia los indicadores socioeconómicos de las tres dimensiones del Índice de Desarrollo Humano (IDH). El análisis de resultados muestra que fueron Baja California Sur, Campeche, Chiapas, Colima, Distrito Federal, Estado de México, Quintana Roo, Tlaxcala y Zacatecas los más eficientes en la generación de bienestar social, haciendo evidente que las entidades con mayores recursos no son necesariamente las que generan mayor bienestar social.

Palabras clave: bienestar social, IDH, DEA y estados de México.

\footnotetext{
* Universidad Michoacana de San Nicolás de Hidalgo, México. Correo-e: cesar126@hotmail.com

** Universidad Michoacana de San Nicolás de Hidalgo, México. Correo-e: franciscoayvar@ hotmail.com

*** Universidad Autónoma de Barcelona, España. Correo-e: victor.gimenez@uab.cat
} 


\section{Introducción}

Durante el periodo 1990-2010, la economía mexicana se caracterizó por incrementos importantes en la población alfabeta, la esperanza de vida al nacer y el Producto Interno Bruto per cápita. Estos aumentos fueron resultado de los esfuerzos gubernamentales ejecutados a través del gasto público y la atracción de inversión, acto que se reflejó en el desarrollo de infraestructura educativa y de salud, contratación de personal (profesores, médicos, enfermeras, etcétera), expansión de la derechohabiencia y la matrícula en las escuelas, así como en el establecimiento de nuevas empresas y el aumento del personal ocupado.

No obstante, aún persisten en el país problemas de desempleo, pobreza, marginación, concentración del bienestar en ciertas áreas geográficas y migración, problemas socioeconómicos que ubican al país en niveles intermedios de la clasificación internacional de desarrollo humano y bienestar social (Inegi, 2013a-e; PNUD, 2013).

El objetivo del trabajo es determinar qué tan eficientes fueron las 32 entidades de la república mexicana en el uso de sus recursos para generar bienestar social durante el periodo 1990-2010. De manera particular se pretende observar, por factor del Índice de Desarrollo Humano -educación, salud e ingreso- qué tan eficientemente se utilizaron los recursos para generar bienestar social.

El bienestar social es la saciedad que experimentan los individuos que componen una comunidad en materia de sus necesidades básicas y superfluas, considerando así aspectos objetivos y subjetivos (Duarte y Jiménez, 2007; Pena-Trapero, 2009). Para la medición del bienestar social se han utilizado tres enfoques: el puramente económico, el basado en las funciones de utilidad y el realizado a través de indicadores sociales sintéticos (Pena-Trapero, 2009). Este último enfoque, que opta por utilizar indicadores sociales sintéticos, ha llevado a la creación de distintos índices, entre los que se encuentra el Índice de Desarrollo Humano (IDH) (Frey y Stutzer, 2002; Zarzosa y Somarriba, 2013). El IDH es un mecanismo de medición del nivel de desarrollo de un país, estado o región a partir de la determinación de su grado de bienestar social, considerando para ello las condiciones de salud, educación e ingreso (León, 2002; Passanante, 2009; PNUD, 2015).

La herramienta que se utilizó para medir la eficiencia fue el Análisis de la Envolvente de Datos (DEA), el cual, basándose en el benchmarking, compara unidades de producción (Farrell, 1957; Charnes et al., 1978; Caves et al., 1982; Bemowski, 1991). Esta técnica recientemente se ha utilizado para medir la calidad de vida, el bienestar económico, el bien- 
estar social y el desarrollo humano (Mahlberg y Obersteiner, 2001; Arcelus et al., 2005; Murias et al., 2009; Yago et al., 2010).

La hipótesis que se considera en la investigación es que muy pocos estados de la república mexicana fueron eficientes en la utilización de sus recursos socioeconómicos para generar bienestar social durante el periodo 1990-2010. Con la finalidad de comprobar la hipótesis se desarrollaron dos modelos DEA. El primero calcula la eficiencia por factor del desarrollo humano. El segundo, tomando en consideración los resultados del primer modelo, determina qué tan eficientemente se utilizaron los recursos de educación, salud e ingreso para generar bienestar social durante 1990-2010.

La investigación se encuentra estructurada en tres apartados: en el primero se abordan los aspectos teóricos del bienestar y del DEA; enseguida se exponen los elementos metodológicos del modelo DEA para calcular la eficiencia en la generación de bienestar social en México y en el tercero se revisan los resultados obtenidos con las mediciones DEA, identificando así las entidades que utilizaron eficientemente sus recursos. Por último, se establecen las conclusiones, en las que se destaca la importancia del uso eficiente de los recursos socioeconómicos para generar bienestar social.

\section{Revisión de literatura}

\subsection{El bienestar social}

Pena-Trapero (2009) establece que existe un conjunto de conceptos estrechamente relacionados con el bienestar social, aunque distintivos, como son el bienestar, la calidad de vida y la felicidad. El bienestar es el sentir de una persona al ver satisfecha todas sus necesidades en materia fisiológica y psicológica en el presente, así como contar con expectativas alentadoras que le sustenten su proyecto de vida (Duarte y Jiménez, 2007). La calidad de vida abarca tres significados: la calidad del entorno en que vivimos, la calidad de acción y el disfrute subjetivo de la vida, haciéndolo un concepto un tanto subjetivo (Setien, 1989; Veenhoven, 1998).

El bienestar social, a pesar de estar íntimamente relacionado con el bienestar y la calidad de vida, tiene una visión más objetiva; de acuerdo con Duarte y Jiménez (2007) y Pena-Trapero (2009), el bienestar social es el conjunto de factores que le permiten al individuo experimentar una saciedad de sus necesidades, desde las más vitales hasta las más superfluas, y que hacen que su existencia posea todos aquellos elementos que dan lugar a la tranquilidad y satisfacción humana. De esta forma, el bienestar social parte del bienestar económico y se desarrolla en concepciones ob- 
jetivas y subjetivas más amplias del bienestar, como son el estar nutrido, tener buena salud, poseer una vivienda digna, contar con acceso a la educación, entre otros (Di Pasquale, 2008; Zarzosa y Somarriba, 2013).

Para la medición del bienestar social se han utilizado tres enfoques diferentes (Sánchez y Rodríguez, 2003; Pena-Trapero, 2009; Zarzosa y Somarriba, 2013):

a) La medición a través del enfoque económico. El fundamento de este tipo de medición está en la asociación del bienestar con la riqueza, utilizando para tal fin el PIB per cápita y el consumo como indicadores bases.

b) El enfoque a través de las funciones de utilidad. Se parte del concepto de satisfacción de necesidades y se recurre a las funciones de utilidad, como mecanismos para determinar la utilidad proporcionada por los bienes y servicios puestos a disposición de los individuos y de la sociedad.

c) La medición del bienestar social con indicadores sociales sintéticos. Parte de la idea de que el bienestar social es un concepto multifacético que sólo puede abarcarse descomponiéndolo en diversas parcelas, cuya integración debería de cubrir su totalidad. Esta visión ha llevado en años recientes a la elaboración de índices resumen o sintéticos que integren en un solo valor los diferentes indicadores, aspectos o dimensiones vinculadas a la problemática.

Actualmente se ha optado por el uso de indicadores sociales sintéticos porque brindan una perspectiva global del bienestar social (Pena-Trapero, 2009). Entre los principales índices sociales sintéticos se encuentran: el Índice de Desarrollo Humano, el Índice Promedio Combinado de Bienestar, el Índice de Bienestar Social, el Índice de Bienestar Social Municipal, el Índice de Desarrollo Sustentable, y el Indicador Medio del Bienestar Social, entre otros (Frey y Stutzer, 2002; Di Pasquale, 2008; Cuenca y Rodríguez, 2010; y Zarzosa y Somarriba, 2013).

El Índice de Desarrollo Humano (IDH) es propuesto por el Programa de Naciones Unidas para el Desarrollo (PNUD), basándose en los postulados de Sen (1991). En esa lógica, el índice considera que los individuos deberán contar con las condiciones básicas del desarrollo humano (disfrutar una vida prolongada y saludable, estar alfabetizado y poseer conocimientos, tener los recursos necesarios para lograr un nivel de vida decente, y participar en la vida de la comunidad), ya que si no se tienen estas oportunidades muchas otras son negadas. De esta forma, el IDH se mide a partir de tres elementos básicos: la educación, la salud y el ingreso, convirtiéndolo en una herramienta que permite evaluar el nivel de bien- 
estar de la sociedad (León, 2002; López-Calva et al., 2004; Passanante, 2009; PNUD, 2015).

Si bien el IDH como indicador social sintético del bienestar social presenta un cuerpo teórico consistente, y se acerca bastante a la complejidad del concepto de bienestar social (Dasgupta y Weale, 1992; Streeten, 1994; Desai, 1993; Haq, 1995; Jasek-Rysdahl, 2001; Di Pasquale, 2008), aún presenta varias críticas técnicas y teóricas al no incluir otras variables vinculadas al bienestar social (Murray, 1991; Trabold-Nubler, 1991; McGillivray, 1991; McGillivray y White, 1993; Dossel y Gounder, 1994; Srinivasan, 1994; Gormley, 1995; Hicks, 1997; Despotis, 2005; y Ravallion, 2012), debido, en gran parte, a la falta de información estadística fiable (Anand y Sen, 2000; Cuenca y Rodríguez, 2010). Entre los aspectos sociales subjetivos y objetivos que se dejan de lado se encuentran los siguientes (Doyal y Gough, 1991; Chasco y Hernández, 2003; Blanco y Díaz, 2005; OCDE, 2014): integración social, aceptación social, contribución social, actualización social, coherencia social, autosuficiencia, equidad, cohesión social, contexto social, así como entorno natural y clima.

\subsection{El Análisis de la Envolvente de Datos (DEA)}

La idea de Farrell (1957), quien explica que para medir la eficiencia de un conjunto de unidades productivas es necesario conocer la función de producción y la frontera de eficiencia, ha podido trasladarse a su aplicación empírica a través de dos metodologías: la estimación de fronteras estocásticas y las mediciones DEA. La primera implica el uso de la econometría y la segunda recurre a algoritmos de programación lineal y al benchmarking. La programación lineal es una técnica pionera en el análisis de las decisiones internas de una empresa sobre la asignación de recursos. La programación lineal representa uno de los avances más importantes en la teoría de la producción, su ventaja principal es de cálculo y se basa en el uso de computadoras, en tanto que el benchmarking se puede definir como la medida de una actuación en comparación con la de las mejores compañías de su clase (Bemowski, 1991; Pinzón, 2003; Serra, 2004).

El DEA es una técnica utilizada para la medición de la eficiencia comparativa de unidades homogéneas. Se parte de los inputs y outputs, este método proporciona un ordenamiento de los agentes, otorgándoles una puntuación de eficiencia relativa. Un agente o DMU (Unidad de Toma de Decisión) es eficiente, es decir, pertenece a la frontera de producción, cuando produce más de algún output sin generar menos del resto y sin consumir más inputs, o bien, cuando utilizando menos de algún input, y no más del resto, genere los mismos productos. De igual forma, los modelos DEA aprovechan el know-how de las DMUs y una vez deter- 
minado quién es eficiente y quién no, busca fijar objetivos de mejora para las segundas, a partir de los logros de las primeras, es decir, realizan un benchmarking de las unidades evaluadas, empleando la información disponible en las propias empresas (Navarro y Torres, 2003).

Existen cuatro principales modelos DEA: el de Rendimientos Constantes a Escala (CRS), el de Rendimientos Variables a Escala (VRS), el aditivo y el multiplicativo. Los modelos DEA pueden tener dos orientaciones: hacia la optimización en la combinación de inputs (modelo inputorientado) para la obtención del output, o hacia la optimización en la producción de outputs (modelo output-orientado) (Charnes et al., 1978; y Banker et al., 1984). Asimismo, el análisis slacks de las variables en los modelos DEA proporciona la dirección en la cual habrán de mejorarse los niveles de eficiencia de las llamadas unidades de toma de decisión DMUs. Es así que un valor output slack representa el nivel adicional de outputs necesarios para convertir una DMU ineficiente en una DMU eficiente. Un valor input slack representa las reducciones necesarias de los correspondientes inputs para convertir una DMU en eficiente (Coelli et al., 2002).

\subsection{El bienestar social y los modelos DEA}

El Análisis de la Envolvente de Datos recientemente ha sido empleado para medir la calidad de vida, el bienestar económico, el bienestar social y el desarrollo humano. Con relación al primer concepto encontramos la obra de Somarriba y Pena-Trapero (2009), quienes emplearon el DEA y otras metodologías para determinar la calidad de vida en Europa, mientras que López-Vizcaíno y Sánchez-Fernández (2009) estudiaron a las comarcas gallegas, en tanto que Murias et al. (2009) usaron este método para la medición del bienestar económico regional de las provincias españolas e italianas. En términos de bienestar social, el DEA ha sido utilizado por diferentes autores, entre los que destacan Hashimoto e Ishikawa (1993) y Hashimoto y Kodama (1997); sobresalen a su vez las publicaciones de Mahlberg y Obersteiner (2001) y Despotis (2005) que utilizan el DEA para construir índices sintéticos, específicamente el IDH. Retomando estas investigaciones, Arcelus et al. (2005) y Yago et al. (2010) buscaron medir la eficiencia de los procesos de transformación de recursos en resultados de desarrollo humano. Otras investigaciones que involucran el DEA, el bienestar social y sus dimensiones son las de Goñi (1998), Miranda y Araya (2003), Pardo y García (2004), Prior y Surroca (2004), Montenegro (2005), Martín (2008), Emrouznejad et al. (2010), Ayaviri y Quispe (2011), entre otros. 


\section{Metodología}

En la presente investigación se decidió utilizar la metodología DEA porque es una técnica que permite identificar la eficiencia por dimensión del IDH, así como cuantificar en un solo indicador global el uso eficiente de los recursos para generar bienestar social, obteniéndose, a partir de las comparaciones interterritoriales entre los 32 estados de la república mexicana, una visión integrada del bienestar social en el país y de la eficiencia para generarlo en el periodo 1990-2010.

La determinación del nivel de eficiencia de los estados de México en la utilización de los recursos en educación, salud e ingreso para generar bienestar social implicó la elaboración de dos modelos DEA. El primero conllevó el cálculo de la eficiencia en el uso de los recursos por dimensión del desarrollo humano para los 32 estados durante el periodo 1990-2010. De esta forma, el primer modelo se sustentó en Rendimientos Variables a Escala (VRS), es decir, cada unidad analizada es comparada con aquéllas de su tamaño y no con todas las unidades presentes en el problema. Estuvo orientado al output, debido a que la finalidad última es maximizar el bienestar social, lo cual implica aumentar el nivel de alfabetismo, esperanza de vida e ingreso per cápita con los recursos que cuentan cada una de las entidades federativas para estos factores. La expresión matemática de este modelo quedó de la siguiente manera:

$$
\begin{gathered}
\operatorname{Max}_{\text {s.a }} \\
\left(\sum_{J=1}^{I} \lambda_{\mathrm{j}} \mathrm{y}_{\mathrm{rj}}\right)-s_{r}^{+}=\varnothing y_{r 0} r=1 \ldots . m \\
\left(\sum_{J=1}^{I} \lambda_{\mathrm{j}} \mathrm{x}_{\mathrm{ij}}\right)-s_{i}^{-}=x_{i 0} i=1 \ldots . m \\
\lambda_{\mathrm{j}}, s_{r}^{+}, s_{i}^{-} \geq 0 ; \emptyset \text { libre de signo }
\end{gathered}
$$

Aquí se supone la existencia de $n D M U$ s, cada una de las cuales puede aplicar $m$ inputs para producir soutputs, asignándole al vector Xij la cantidad de input $i$ utilizado por la $D M U j$, mientras que el vector $Y r j$ representa la cantidad de output $r$ producido por la $D M U j$. La variable $(\lambda j)$ indica el peso de la $D M U z$ en la construcción de la unidad virtual de referencia respecto de la $D M U j$, que puede ser obtenida por la com- 
binación lineal del resto de $D M U s$. Si dicha unidad virtual no puede ser conseguida, entonces la $D M U z$, para la que resuelve el sistema, se considerará eficiente. El escalar $(\varphi)$ representa la mayor expansión radial de todos los outputs producidos por la unidad evaluada, variando su rango entre 1 y $\infty$, de forma que tomará valor unitario cuando la unidad sea eficiente y valores superiores a 1 cuando sea ineficiente (Navarro, 2005).

Los outputs del primer modelo son los tres componentes del IDH de las entidades federativas de México. La razón de haber escogido estos outputs se encuentra en la representatividad teórica que tienen las tres dimensiones para explicar el bienestar social de un país o región (Baquero, 2004; Arcelus et al., 2005; Despotis, 2005; Ramos y Silber, 2005; Yago et al., 2010; y Jahanshahloo et al., 2011). La información estadística de esperanza de vida al nacer, alfabetismo, matriculación y PIB per cápita fue posible obtenerla a través de las bases estadísticas del Instituto Nacional de Estadística y Geografía de México (Inegi), la Secretaría de Educación Pública de México (SEP), el Consejo Nacional de Población (Conapo), la Secretaría de Salud de México, así como de los Informes sobre Desarrollo Humano del PNUD.

La selección de inputs del primer modelo, en su primera etapa, se sustentó en los pilares teóricos que explican el comportamiento de los componentes del IDH. En tal sentido, se analizaron los postulados de Baquero (2004), Arcelus et al. (2005), Despotis (2005), Montenegro (2005), Blancas y Domínguez (2010), Blancard y Hoarau (2011), Yago et al. (2010), Emrouznejad et al. (2010), Jahanshahloo et al. (2011) y PNUD (2015), determinando que los indicadores que explican el comportamiento de las dimensiones del desarrollo humano, son:

a) Educación (matriculación y alfabetismo). Los indicadores que explican esta dimensión son: gasto en educación pública, niños que llegan al quinto grado, relación mujeres/hombres en alfabetismo, relación mujeres/hombres en matriculación, PIB per cápita, total de profesores, aulas y escuelas disponibles.

b) Salud (esperanza de vida al nacer). Los indicadores que explican la dimensión son: población con servicios de saneamiento, población con fuentes de agua, población con acceso a medicamentos, niños de un año inmunizados, partos atendidos por personal de salud, médicos, gasto en salud, habitantes desnutridos, personas con VIH/ sida, consumo de cigarrillos, tasa de mortalidad de menores de un año, tasa de mortalidad de menores de cinco años, tasa de mortalidad materna, PIB per cápita y camas disponibles en los hospitales.

c) Ingreso (PIB per cápita). Los indicadores que explican esta dimensión son: cambio medio anual del índice de precios al consumidor, 
índice de desigualdad, exportaciones, importaciones, inversión extranjera directa, total de servicio de la deuda, asistencia para el desarrollo, gasto público, consumo de electricidad per cápita, proporción de población que usa internet, grado de escolarización, población económicamente activa, personal ocupado, unidades económicas, formación bruta de capital, remuneraciones y salario.

Dada la disponibilidad de información estadística para los estados de la república mexicana, la cantidad de indicadores se vio reducida. Con estos datos se procedió a realizar un análisis factorial, ${ }^{1}$ empleando como método de extracción los componentes principales. De esta forma se determinó, en primera instancia, una matriz de correlaciones para cada una de las dimensiones del IDH. Posteriormente, y con valores superiores al 0,6 en la prueba de KMO y niveles de significancia menores al 0,05 en la prueba de Bartlett, se corroboró la factibilidad de efectuar el análisis factorial. Finalmente, se llevaron a cabo los ensayos factoriales y con los resultados de la matriz de componentes, por dimensión del IDH, se determinó que los modelos a realizar en la investigación quedaran de la siguiente manera:

a) Educación (alfabetismo). Los inputs que explican esta dimensión son: profesores y aulas disponibles.

b) Salud (esperanza de vida al nacer). Los inputs que explican esta dimensión son: médicos disponibles y población con derechohabiencia.

c) Ingreso (PIB per cápita). Los inputs que explican esta dimensión son: gasto público, grado de escolarización y personal ocupado.

Una vez efectuado el análisis factorial, y determinado por dimensión del desarrollo humano los inputs del modelo DEA, se llevaron a efecto pruebas econométricas con datos panel, mínimos cuadrados ordinarios y efectos fijos (dado los resultados del Test de Hausman), con la finalidad de establecer el grado de correlación que tienen los inputs con los outputs por dimensión del IDH. Los resultados de estas pruebas nos permitieron concluir que los insumos de producción, reflejados en los inputs, afectan

${ }^{1}$ El análisis factorial es una técnica de reducción de datos que sirve para encontrar grupos homogéneos de variables a partir de un conjunto numeroso de variables. Dichos grupos homogéneos se forman con variables que correlacionan mucho entre sí, procurando que unos grupos sean independientes de otros. El análisis factorial consta de cuatro etapas: el cálculo de una matriz de correlaciones, capaz de expresar la variabilidad conjunta de todas la variables; la extracción del número óptimo de factores; la rotación de la solución para facilitar su interpretación; y la estimación de las puntuaciones de los sujetos en las nuevas dimensiones (Espejel et al., 2004; Akinyokun y Uzoka, 2007; Montoya, 2007). 
directamente los outputs expresados en los niveles de alfabetismo, esperanza de vida al nacer y el PIB per cápita.

El segundo modelo se construyó a partir de las eficiencias obtenidas por dimensión del primer modelo, es decir, calcula qué entidad fue la más eficiente en la generación de desarrollo humano. Se orienta al input, ya que en este caso lo que interesa es identificar a las $D M U_{s}$ que con menos insumos (educación, salud e ingreso) generan más output, que en este caso es la unidad (1) ya que representa el valor máximo alcanzable en los modelos DEA. Este modelo se basó en Rendimientos Constantes a Escala (CRS) con lo cual cada unidad analizada es comparada con todas las unidades presentes en el problema, esto es factible dado que los valores ya se encuentran normalizados, producto del modelo anterior. La expresión matemática de este segundo modelo, es:

Eficiencia Total $=\frac{1}{\text { Ef.en Educación }+ \text { Ef. en Salud }+ \text { Ef.en ingreso }}$

Como se mencionó, el segundo modelo DEA se basa en CCR y con orientación input, siendo su expresión matemática la siguiente:

$$
\begin{gathered}
\operatorname{Min} \varnothing \\
s . a \\
\left(\sum_{J=1}^{I} \lambda_{\mathrm{j}} \mathrm{y}_{\mathrm{rj}}\right)-s_{r}^{+}=y_{r 0} r=1 \ldots . m \\
\left(\sum_{J=1}^{I} \lambda_{\mathrm{j}} \mathrm{x}_{\mathrm{ij}}\right)+s_{i}^{-}=\emptyset x_{i 0} i=1 \ldots . m \\
\lambda_{\mathrm{j}}, s_{r}^{+}, s_{i}^{-} \geq 0 ; \emptyset \text { libre de signo }
\end{gathered}
$$

El escalar $\varphi$ representa la menor expansión radial de todos los inputs consumidos por la unidad evaluada, variando su rango entre 0 y 1 , de forma que tomará valor unitario cuando la unidad sea eficiente y valores menores a 1 cuando sea ineficiente (Navarro, 2005 y Giménez, 2000).

\section{Resultados}

A continuación se presentan los resultados obtenidos por dimensión del IDH, y en su conjunto, para cada uno de los estados de la república mexicana, durante el periodo 1990-2010. 


\subsection{La eficiencia en el factor educación}

El cuadro 1 del anexo muestra que los niveles de eficiencia en la utilización de los recursos educativos (profesores y aulas disponibles) para generar bienestar en educación (personas que saben leer y escribir) de los estados mexicanos son bajos, ya que 23 entidades fueron clasificadas como ineficientes. Las entidades consideradas como eficientes durante el periodo 1990-2010 fueron Baja California Sur, Estado de México, Nuevo León y Quintana Roo. Asimismo, destacan Colima, Chihuahua, Distrito Federal, Tabasco y Tlaxcala por ser eficientes al menos en un par de años. Por otro lado, las entidades clasificadas como más ineficientes fueron San Luis Potosí, Oaxaca, Guerrero, Chiapas y Durango que deberán incrementar en promedio $56 \%$ su población alfabeta con la cantidad de profesores y aulas que actualmente tienen disponibles. El esfuerzo de alfabetización de la población en estos estados, además de implicar el mejor aprovechamiento de los recursos mencionados, requiere de un mejor uso del gasto en educación así como el diseño y ejecución de políticas públicas que aumenten la calidad, la matriculación y la eficiencia terminal en los distintos niveles educativos, que a la par disminuyan la desigualdad y el rezago educativo (Fernández y Blanco, 2004; Acosta, 2010; Arzate, 2011; Scott, 2011; Narro et al., 2013; Barba, 2015; y Rojas, 2015).

\subsection{La eficiencia en el factor salud}

Los resultados de eficiencia en la dimensión salud del cuadro 2 del anexo evidencian que la mayoría de los estados de la república mexicana utilizaron eficientemente sus recursos (médicos y derechohabiencia) para generar bienestar en salud (esperanza de vida al nacer). Las entidades de Baja California Sur, Chiapas, Colima, Nayarit, Oaxaca, Tlaxcala y Zacatecas fueron las más eficientes durante todo el periodo de estudio, mientras que Aguascalientes, Distrito Federal, Quintana Roo y Tabasco solamente en algunos años. Por otro lado, los estados de Puebla, Sonora, Hidalgo y Guerrero fueron los más ineficientes por lo que requieren aumentar la esperanza de vida al nacer en $2 \%$, en promedio, con los insumos que poseen. El incremento de la esperanza de vida al nacer en estas entidades, además de necesitar el uso eficiente de los recursos, requiere de mayores esfuerzos en la orientación del gasto público en salud, en equipamiento, campañas de vacunación y demás acciones preventivas para disminuir la tasa de mortalidad, así como el planteamiento y ejercicio de políticas públicas que fomenten la universalidad en el acceso al sistema de salud (Acosta, 2010; Scott, 2011; Narro et al., 2013; Barba, 2015; Valencia, 2015; Rojas, 2015). 


\subsection{La eficiencia en el factor ingreso}

En el factor ingreso los niveles de ineficiencia son altos, 22 estados fueron ineficientes en la utilización de sus recursos (gasto público, grado de escolarización y personal ocupado) para generar bienestar económico (PIB per cápita). Las entidades de Baja California Sur, Campeche, Chiapas, Colima, Distrito Federal, Quintana Roo y Zacatecas fueron las más eficientes durante el periodo 1990-2010, mientras que Guerrero, Oaxaca, Tlaxcala y Yucatán solamente en algunos ańos, en tanto que Durango, Puebla, Tabasco y Veracruz destacan como las más ineficientes, por lo que deberán hacer crecer su nivel de ingreso per cápita en 139\% con los recursos que poseen. Dicha ampliación demanda de forma adicional el uso productivo de la inversión pública y privada para la creación de empleos, así como la elaboración y cumplimiento de políticas públicas que permitan disminuir la concentración del ingreso, la pobreza y la marginación (Acosta, 2010; Rojas, 2010 y 2015; Scott, 2011; Narro et al., 2013 y Barba, 2015) (cuadro 3 del anexo).

\subsection{La eficiencia en la generación de bienestar social}

Al considerar los tres elementos del desarrollo humano (educación, salud e ingreso), los resultados del modelo de eficiencia establecen que 16 estados son ineficientes en la utilización de sus recursos para generar bienestar social; entre ellos destacan Veracruz, Sonora, San Luis Potosí y Guerrero como entidades que tienen más dificultades que otras para utilizar correctamente sus recursos para generar bienestar en su población, sobre todo los concernientes a la dimensión educación e ingreso. Baja California Sur, Campeche, Chiapas, Colima, Distrito Federal, Estado de México, Quintana Roo, Tlaxcala y Zacatecas se encuentran dentro de los estados más eficientes durante todo el periodo de estudio (cuadro 4 del anexo).

Al comparar los resultados de eficiencia de las tres dimensiones con el Índice de Desarrollo Humano del PNUD (2011), se identificó que los estados clasificados con mayor nivel de IDH (Baja California, Baja California Sur, Chihuahua, Distrito Federal, Nuevo León y Quintana Roo) no necesariamente fueron los más eficientes en la utilización de sus recursos para generar bienestar social. Sin embargo, en el caso de Baja California Sur, Distrito Federal y Quintana Roo, los altos niveles de desarrollo humano se vinculan directamente a la utilización eficiente de los recursos (cuadros 1 a 4 del anexo).

Los resultados del estudio permiten concluir que el uso eficiente de los recursos incide directamente en el grado de bienestar de la población, relación que ya había sido expuesta por autores como Mahlberg y Obers- 
teiner (2001) en su análisis de 174 países; Baquero (2004) en su investigación sobre Venezuela; Despotis (2005) en su estudio de los países del Asia Pacífico; Arcelus et al. (2005) en su análisis de diversos países; Lee et al. (2006) en su investigación de 27 países de la región Asia Pacífico; Reig y Soler (2009) en su estudio del caso español; Yago et al. (2010) en su análisis de los estados mexicanos; Shetty y Pakkala (2010) en su investigación sobre los estados de la India; Blancard y Hoarau (2011) en su diagnóstico internacional; y Wu et al. (2014) en su estudio sobre 19 países de la OCDE.

De esta forma, los resultados de la presente investigación coincide con las conclusiones expresadas en otros estudios, así como con los postulados teóricos que ven en la consolidación del bienestar social un aspecto fundamental para acceder a mayores niveles de desarrollo (Agudelo y Cuervo, 1999; Millán, 2001; Sánchez y Rodríguez, 2003; Zarzosa y Somarriba, 2013 y Sen, 1991, 2001 y 2014).

\section{Conclusiones}

El bienestar social es el conjunto de factores que le permite al individuo experimentar una saciedad de sus necesidades objetivas y subjetivas, y que hacen que su existencia sea tranquila y satisfactoria. La medición del bienestar social en los últimos años se ha orientado a la integración de indicadores sociales sintéticos, entre los que destaca el IDH, índice que a pesar de sus críticas es uno de los indicadores que más se acerca a la complejidad del concepto de bienestar social.

Tomando en consideración los postulados teóricos del PNUD, se desarrolló un modelo haciendo uso de la técnica DEA para determinar qué tan eficientes fueron las entidades de México en el uso de los recursos para generar bienestar social. El modelo se elaboró en dos etapas, la primera con rendimientos variables a escala y orientado al output. Los outpute inputs del modelo quedaron establecidos de la siguiente manera: a) Educación, el output fue alfabetismo y los inputs los profesores y las aulas disponibles; b) Salud, el output fue la esperanza de vida al nacer y los inputs los médicos disponibles y la población con derechohabiencia; c) Ingreso, el output fue el PIB per cápita y los inputs el gasto público, el grado de escolarización y el personal ocupado. La segunda etapa del modelo se elaboró bajo rendimiento constante a escala y con orientación al input, determinando para ello a la unidad (1) como output y como inputs los resultados de eficiencia de las tres dimensiones.

En el factor educación las entidades clasificadas como las más eficientes para el periodo 1990-2010 fueron: Baja California Sur, Estado de México, Nuevo León, Quintana Roo y Tlaxcala. Por lo que se refiere al 
factor salud, los estados de Baja California Sur, Chiapas, Colima, Nayarit, Oaxaca, Tlaxcala y Zacatecas se encontraron dentro de los más eficientes en la generación de bienestar. En el factor ingreso los estados de Baja California Sur, Campeche, Chiapas, Colima, Distrito Federal, Quintana Roo y Zacatecas alcanzaron las mayores eficiencias.

Al conjuntar los resultados de eficiencia de las dimensiones del IDH en un indicador sintético se tiene que las entidades de Baja California Sur, Campeche, Chiapas, Colima, Distrito Federal, Estado de México, Quintana Roo, Tlaxcala y Zacatecas fueron las más eficientes en la generación de bienestar social durante el periodo 1990-2010, haciendo evidente que los estados con mayor Índice de Desarrollo Humano en general no siempre fueron los más eficientes en la utilización de sus recursos.

Dentro de las principales limitaciones de la investigación se encuentran el que no se incluyen a otras variables del bienestar social más allá de las utilizadas por el IDH, y que no se profundiza en los elementos que pueden hacer del DEA una técnica más robusta como método de agregación para la integración de indicadores sociales sintéticos. Por último, las líneas de investigación futuras podrían orientarse a superar las debilidades de la investigación en los siguientes términos: a) incorporar en los modelos DEA un enfoque de análisis dinámico y de inclusión de variables intermedias, no discrecionales y no deseadas; $b$ ) explorar los factores institucionales y regionales que afectan la eficiencia en la generación de bienestar; $y, c)$ diseñar políticas públicas que, contemplando el uso eficiente de los recursos, permitan acceder a un mayor nivel de bienestar social.

\section{Agradecimientos}

Francisco Javier Ayvar Campos agradece el apoyo y financiamiento del Consejo Nacional de Ciencia y Tecnología (Conacyt) de México para la realización de esta investigación, en el marco de la Estancia de Investigación Posdoctoral en el Extranjero 2015. 


\section{Anexo}

\section{Cuadro 1}

Cálculo de la eficiencia del factor educación, 1990-2010

\begin{tabular}{|c|c|c|c|c|c|}
\hline$D M U$ & 1990 & 1995 & 2000 & 2005 & 2010 \\
\hline Aguascalientes & 1,1569 & 1,2008 & 1,1521 & 1,1393 & 1,1337 \\
\hline Baja California & 1,1196 & 1,0483 & 1,1123 & 1,1427 & 1,1641 \\
\hline Baja California Sur & 1 & 1 & 1 & 1 & 1 \\
\hline Campeche & 1,2855 & 1,2416 & 1,1846 & 1,1103 & 1,0098 \\
\hline Chiapas & 1,2648 & 1,3681 & 1,3966 & 1,3613 & 1,3254 \\
\hline Chihuahua & 1,019 & 1,0272 & 1,0222 & 1,0553 & 1,0249 \\
\hline Coahuila & 1,2231 & 1,2 & 1,1587 & 1,1188 & 1,1457 \\
\hline Colima & 1,3763 & 1,2201 & 1,1856 & 1 & 1 \\
\hline Distrito Federal & 1 & 1,2831 & 1,3825 & 1,3538 & 1,3981 \\
\hline Durango & 1,3372 & 1,4058 & 1,3549 & 1,282 & 1,3333 \\
\hline Guanajuato & 1,0478 & 1,0601 & 1,1238 & 1,117 & 1,1379 \\
\hline Guerrero & 1,5215 & 1,5576 & 1,4584 & 1,4866 & 1,4366 \\
\hline Hidalgo & 1,2013 & 1,2393 & 1,2885 & 1,3292 & 1,3185 \\
\hline Jalisco & 1,0553 & 1,1175 & 1,18 & 1,1453 & 1,193 \\
\hline México & 1 & 1 & 1 & 1 & 1 \\
\hline Michoacán & 1,1583 & 1,2065 & 1,2215 & 1,2494 & 1,2457 \\
\hline Morelos & 1,1369 & 1,0828 & 1,057 & 1,0864 & 1,1164 \\
\hline Nayarit & 1,3385 & 1,2941 & 1,2283 & 1,2415 & 1,2637 \\
\hline Nuevo León & 1,0544 & 1,0772 & 1 & 1 & 1 \\
\hline Oaxaca & 1,1792 & 1,3007 & 1,3055 & 1,4013 & 1,3724 \\
\hline Puebla & 1 & 1,1932 & 1,1954 & 1,2011 & 1,1933 \\
\hline Querétaro & 1,1365 & 1,0823 & 1,0208 & 1,0189 & 1,0231 \\
\hline Quintana Roo & 1 & 1 & 1,001 & 1,0297 & 1 \\
\hline San Luis Potosí & 1,3895 & 1,3658 & 3,6938 & 1,2958 & 1,2762 \\
\hline Sinaloa & 1,2525 & 1,2563 & 1,223 & 1,2339 & 1,2021 \\
\hline Sonora & 1,1725 & 1,1185 & 1,105 & 1,0988 & 1,0721 \\
\hline Tabasco & 1,1132 & 1,1489 & 1,0708 & 1 & 1,0494 \\
\hline Tamaulipas & 1,2048 & 1,1258 & 1,0687 & 1,0626 & 1,0398 \\
\hline Tlaxcala & 1 & 1,1257 & 1,0663 & 1 & 1 \\
\hline Veracruz & 1,2423 & 1,3804 & 1,223 & 1,206 & 1,238 \\
\hline Yucatán & 1,2245 & 1,3014 & 1,2851 & 1,2175 & 1,1876 \\
\hline Zacatecas & 1,1498 & 1,1553 & 1,1592 & 1,1908 & 1,2191 \\
\hline
\end{tabular}

Fuente: Elaboración propia con base en los datos publicados por el Inegi (2013a y e) y la SEP (2013a y b), y utilizando el programa EMS. 


\section{Cuadro 2}

\section{Cálculo de la eficiencia del factor salud, 1990-2010}

\begin{tabular}{|c|c|c|c|c|c|}
\hline$D M U$ & 1990 & 1995 & 2000 & 2005 & 2010 \\
\hline Aguascalientes & 1 & 1,0035 & 1,0082 & 1,0103 & 1,0102 \\
\hline Baja California & 1,0031 & 1,0083 & 1,0075 & 1,0026 & 1,0025 \\
\hline Baja California Sur & 1 & 1 & 1 & 1 & 1 \\
\hline Campeche & 1,029 & 1,0303 & 1,0244 & 1,0131 & 1,013 \\
\hline Chiapas & 1 & 1 & 1 & 1 & 1 \\
\hline Chihuahua & 1,0096 & 1,0096 & 1,0079 & 1,0053 & 1,005 \\
\hline Coahuila & 1,001 & 1,0096 & 1,0115 & 1,0185 & 1,0189 \\
\hline Colima & 1 & 1 & 1 & 1 & 1 \\
\hline Distrito Federal & 1 & 1 & 1,0016 & 1,0024 & 1,0026 \\
\hline Durango & 1,0217 & 1,0134 & 1,0108 & 1,0207 & 1,0211 \\
\hline Guanajuato & 1,0133 & 1,0088 & 1,007 & 1,0089 & 1,0085 \\
\hline Guerrero & 1,0555 & 1,0351 & 1,0284 & 1,0342 & 1,0209 \\
\hline Hidalgo & 1,0427 & 1,0197 & 1,0138 & 1,0154 & 1,0154 \\
\hline Jalisco & 1,013 & 1,0053 & 1,0076 & 1,0101 & 1,0103 \\
\hline México & 1,0037 & 1,0032 & 1,0043 & 1,0031 & 1,0031 \\
\hline Michoacán & 1,0066 & 1,0083 & 1,0075 & 1,0165 & 1,0161 \\
\hline Morelos & 1,0224 & 1,0058 & 1,0034 & 1,0012 & 1,0019 \\
\hline Nayarit & 1 & 1 & 1 & 1,0113 & 1,0127 \\
\hline Nuevo León & 1,0068 & 1,0037 & 1,0054 & 1,0104 & 1,0115 \\
\hline Oaxaca & 1 & 1 & 1 & 1 & 1,0069 \\
\hline Puebla & 1,0312 & 1,026 & 1,0202 & 1,0115 & 1,0099 \\
\hline Querétaro & 1,0243 & 1,0218 & 1,0203 & 1,0102 & 1,0095 \\
\hline Quintana Roo & 1,0301 & 1,013 & 1,0085 & 1 & 1 \\
\hline San Luis Potosí & 1,0254 & 1,0164 & 1,0118 & 1,0118 & 1,0119 \\
\hline Sinaloa & 1,0202 & 1,0042 & 1,0067 & 1,0182 & 1,0196 \\
\hline Sonora & 1,0361 & 1,0274 & 1,022 & 1,0214 & 1,0195 \\
\hline Tabasco & 1 & 1 & 1,0021 & 1,0153 & 1,0154 \\
\hline Tamaulipas & 1,0206 & 1,0145 & 1,011 & 1,0043 & 1,0034 \\
\hline Tlaxcala & 1 & 1 & 1 & 1 & 1 \\
\hline Veracruz & 1,0278 & 1,0219 & 1,019 & 1,0289 & 1,0275 \\
\hline Yucatán & 1,0272 & 1,0168 & 1,0155 & 1,0119 & 1,0121 \\
\hline Zacatecas & 1 & 1,0143 & 1 & 1 & 1,0038 \\
\hline
\end{tabular}

Fuente: Elaboración propia con base en datos publicados por el Inegi (2013 a), el Conapo (2013) y la Secretaría de Salud (2013a y b) y utilizando el programa EMS. 


\section{Cuadro 3}

Cálculo de la eficiencia del factor ingreso, 1990-2010

\begin{tabular}{|c|c|c|c|c|c|}
\hline$D M U$ & 1990 & 1995 & 2000 & 2005 & 2010 \\
\hline Aguascalientes & 2,4886 & 1,4138 & 1,1541 & 4,8519 & 7,6018 \\
\hline Baja California & 2,1319 & 1,4791 & 1,3063 & 6,885 & 9,9964 \\
\hline Baja California Sur & 1 & 1 & 1 & 1 & 1 \\
\hline Campeche & 1 & 1 & 1 & 1 & 1 \\
\hline Chiapas & 1 & 1 & 1 & 1 & 1 \\
\hline Chihuahua & 1,973 & 1,491 & 1,2501 & 7,2412 & 10,2054 \\
\hline Coahuila & 2,4848 & 1,4251 & 1,3345 & 5,7537 & 7,6644 \\
\hline Colima & 1 & 1 & 1 & 1 & 1 \\
\hline Distrito Federal & 1 & 1 & 1 & 3,5549 & 4,7047 \\
\hline Durango & 2,8042 & 1,1439 & 2,1108 & 8,8183 & 11,4659 \\
\hline Guanajuato & 1,9313 & 1,9136 & 1,4592 & 5,9303 & 8,1377 \\
\hline Guerrero & 1,3674 & 1 & 1,7747 & 5,5014 & 9,7972 \\
\hline Hidalgo & 1,0029 & 2,4069 & 2,1886 & 8,6471 & 11,8018 \\
\hline Jalisco & 2,1152 & 2,1404 & 1,8362 & 7,6191 & 10,1201 \\
\hline México & 2,586 & 2,7531 & 2,7043 & 11,4183 & 15,0757 \\
\hline Michoacán & 2,1556 & 2,4033 & 1,9211 & 5,7548 & 7,8044 \\
\hline Morelos & 1,9298 & 2,0835 & 2,0309 & 10,0079 & 14,8878 \\
\hline Nayarit & 2,5363 & 2,6975 & 2,8096 & 7,9953 & 15,2588 \\
\hline Nuevo León & 1,474 & 1,2393 & 1,0812 & 4,1218 & 5,2578 \\
\hline Oaxaca & 1,5127 & 1,5692 & 1 & 1 & 4,5269 \\
\hline Puebla & 2,6928 & 2,5566 & 2,003 & 8,6537 & 12,2344 \\
\hline Querétaro & 2,4771 & 1,6756 & 1,4131 & 6,8263 & 8,7567 \\
\hline Quintana Roo & 1 & 1 & 1 & 5,7953 & 8,0523 \\
\hline San Luis Potosí & 2,4995 & 2,4846 & 2,092 & 8,6359 & 11,7025 \\
\hline Sinaloa & 2,284 & 2,5592 & 2,37 & 9,5026 & 11,5188 \\
\hline Sonora & 2,3664 & 1,5727 & 1,5151 & 7,1632 & 9,3023 \\
\hline Tabasco & 2,8119 & 2,8825 & 2,7068 & 5,2822 & 5,0748 \\
\hline Tamaulipas & 2,5461 & 1,8483 & 1,6543 & 6,4267 & 8,3746 \\
\hline Tlaxcala & 4,2059 & 1 & 2,7631 & 7,4137 & 19,0698 \\
\hline Veracruz & 2,8679 & 2,3279 & 2,1488 & 7,3388 & 8,7 \\
\hline Yucatán & 1,6317 & 1 & 1 & 7,4689 & 10,6056 \\
\hline Zacatecas & 1 & 1 & 1 & 1 & 1 \\
\hline
\end{tabular}

Fuente: Elaboración propia con base en datos publicados por el Inegi (2013 a, b, c y d) y utilizando el programa EMS. 


\section{Cuadro 4}

\section{Cálculo de la eficiencia en la generación de bienestar social, 1990-2010}

\begin{tabular}{|c|c|c|c|c|c|}
\hline$D M U$ & 1990 & 1995 & 2000 & 2005 & 2010 \\
\hline Aguascalientes & 1 & 0,997 & 0,992 & 0,990 & 0,989 \\
\hline Baja California & 0,997 & 0,992 & 0,994 & 0,997 & 0,996 \\
\hline Baja California Sur & 1 & 1 & 1 & 1 & 1 \\
\hline Campeche & 1 & 1 & 1 & 1 & 1 \\
\hline Chiapas & 1 & 1 & 1 & 1 & 1 \\
\hline Chihuahua & 0,991 & 1 & 1 & 0,993 & 0,994 \\
\hline Coahuila & 0,999 & 0,991 & 0,989 & 0,983 & 0,982 \\
\hline Colima & 1 & 1 & 1 & 1 & 1 \\
\hline Distrito Federal & 1 & 1 & 1 & 1 & 1 \\
\hline Durango & 0,979 & 0,987 & 0,989 & 0,980 & 0,979 \\
\hline Guanajuato & 0,987 & 0,991 & 0,993 & 0,992 & 0,992 \\
\hline Guerrero & 0,947 & 1 & 0,972 & 0,967 & 0,970 \\
\hline Hidalgo & 0,998 & 0,981 & 0,986 & 0,985 & 0,986 \\
\hline Jalisco & 0,987 & 0,995 & 0,993 & 0,991 & 0,990 \\
\hline México & 1 & 1 & 1 & 1 & 1 \\
\hline Michoacán & 0,994 & 0,992 & 0,993 & 0,984 & 0,984 \\
\hline Morelos & 0,978 & 0,994 & 0,997 & 0,998 & 0,998 \\
\hline Nayarit & 1 & 1 & 1 & 0,990 & 0,987 \\
\hline Nuevo León & 0,993 & 0,996 & 0,995 & 1 & 1 \\
\hline Oaxaca & 1 & 1 & 1 & 1 & 0,986 \\
\hline Puebla & 1 & 0,998 & 0,980 & 0,989 & 0,990 \\
\hline Querétaro & 0,976 & 0,979 & 0,980 & 0,990 & 0,990 \\
\hline Quintana Roo & 1 & 1 & 1 & 1 & 1 \\
\hline San Luis Potosí & 0,975 & 0,984 & 0,988 & 0,989 & 0,988 \\
\hline Sinaloa & 0,980 & 0,996 & 0,993 & 0,983 & 0,981 \\
\hline Sonora & 0,965 & 0,973 & 0,979 & 0,979 & 0,980 \\
\hline Tabasco & 1 & 1 & 0,998 & 0,989 & 1 \\
\hline Tamaulipas & 0,980 & 0,986 & 0,989 & 0,997 & 0,998 \\
\hline Tlaxcala & 1 & 1 & 1 & 1 & 1 \\
\hline Veracruz & 0,973 & 0,979 & 0,981 & 0,972 & 0,973 \\
\hline Yucatán & 0,973 & 1 & 1 & 0,988 & 0,988 \\
\hline Zacatecas & 1 & 1 & 1 & 1 & 1 \\
\hline
\end{tabular}

Fuente: elaboración propia con base en los cuadros 1 a 3 del anexo y utilizando el programa EMS. 


\section{Fuentes consultadas}

Acosta, Félix (2010), "La evaluación de la política social en México: avances recientes, tareas pendientes y dilemas persistentes", $\mathrm{Pa}$ peles de población, 16 (64), Universidad Autónoma del Estado de México, Toluca, pp. 155-188.

Agudelo, Germán y John Cuervo (1999), “Crítica a las bases éticas de la teoría neoclásica en la propuesta del bienestar social de Amartya Sen”, Lecturas de economía, núm. 51, Universidad de Antioquía, Antioquía, pp. 111-148.

Akinyokun, O. Charles y F. Uzoka (2007), "Factor analysis of the effects of academic staff profile on the investment portfolio of a University", International Journal of the Computer, the Internet and Management, 15 (1), Srisakdi Charmonman Institute, Bangkok Yai, pp. 51-62.

Anand, Sudhir y Amartya Sen (2000), "The income component of the human development index", Journal of human development", 1 (1), Taylor \& Francis, pp. 83-106.

Arcelus, Francisco, Kanhaiya Lai Sharma y Gopalan Srinivasan (2005), "The human development index adjusted for efficient resource utilization", research paper No. 2005/08, United Nations University-World Institute for Development Economics Research, <http://www.wider.unu.edu/publications/workingpapers/research-papers/2005/en_GB/rp2005-08/>, 15 de noviembre de 2015 .

Arzate, Jorge (2011), "Evaluación analítica de políticas educativas compensatorias en México: el caso de los programas de lucha contra la pobreza, 1988-2011", Revista Mexicana de Investigación Educativa, 16 (51), Consejo Mexicano de Investigación Educativa, A. C., México, pp. 1055-1085.

Ayaviri, Dante y Gabith Quispe (2011), “Medición de la eficiencia asignativa mediante el análisis envolvente de datos en los municipios de Bolivia: caso municipios de Potosí”, Perspectivas, 14 (28), Universidad Católica Boliviana San Pablo, Cochabamba, pp. 137-169. 
Banker, Rajiv, A. Charnes y William Cooper (1984), "Some models for estimating technical and scale inefficiencies in data envelopment analysis", Management Science, 30 (9), Informs Pubs online, pp. 1078-1092.

Baquero de Jiménez, Nancy (2004), "Una aproximación metodológica para el cálculo del IDH mediante el Análisis Envolvente de Datos: Índice de bienestar", ponencia presentada en el III Congreso Colombiano y I Conferencia Andina de Investigación de Operaciones, marzo 2004, Cartagena, <http://prof.usb.ve/nbaquero/ ESTIMACION\%20IDH\%20VIA\%20DEA.pdf>, 15 de noviembre del 2015.

Barba, Carlos (2015), "Los enfoques latinoamericanos sobre la política social: más allá del consenso de Washington", Espiral. Estudios sobre Estado y sociedad, 11 (31), Universidad de Guadalajara, Guadalajara, pp. 85-130.

Bemowski, Karen (1991), “The benchmarking bandwagon”, Quality Progress, 24 (1), American Society for Quality Control, Milwaukee, pp. 19-24.

Blanco, Amalio y Darío Díaz (2005), "El bienestar social: su concepto y medición”, Psicothema, 17 (4), Universidad de Oviedo, Oviedo, pp. 582-589.

Blancard, Stéphane y Jean François Hoarau (2011), "Optimizing the new formulation of the United Nations human development index: an empirical view from data envelopment analysis", Economics Bulletin, 31(1), Elsevier, Amsterdam, pp. 989-1003.

Blancas, Francisco y Mónica Domínguez (2010), “Un indicador sintético DEA para la medición de bienestar desde una perspectiva de género", Revista Investigación Operacional, 31 (3), Universidad de La Habana, La Habana, pp. 255-239.

Caves, Douglas, Laurits Christensen y Erwin Diewert (1982), “The economic theory of index numbers and the measurement of input, output, and productivity", Econometrica, 50 (6), Econometric Society, pp. 1393-1414. 
Charnes, Abraham, William Cooper y Eduardo Rhodes (1978), "Measuring efficiency of decision making units", European Journal of Operational Research, 2 (6), Elsevier, Amsterdam, pp. 429-444.

Chasco, Coro e Inve Hernández (2003), "Medición del bienestar social provincial a través de indicadores objetivos", XVII Congreso de la Asociación de Economía Aplicada, junio, España. <http://www. asepelt.org/ficheros/File/Anales/2003\%20-\%20Almeria/asepeltPDF/111.PDF>, 12 de noviembre de 2015.

Coelli, Tim, Sanzidur Rahman y Colin Thirtle (2002), "Technical, allocative, cost and scale efficiencies in Bangladesh rice cultivation: A non-parametric approach", Journal of Agricultural Economics, 53 (3), Wiley, pp. 607-626.

Conapo (Consejo Nacional de Población) (2013), "Indicadores demográficos, 1990-2050”, Conapo, México, <http://www.conapo. gob.mx/es/conapo/proyecciones_datos>, 15 de noviembre de 2015.

Cuenca, Eduardo y José Antonio Rodríguez (2010), “Medición de las disparidades entre indicadores asociados al bienestar social en los países menos adelantados (PMA) de Asia", Revista de Economía Mundial, núm. 25, Sociedad de Economía Mundial, Huelva, pp. 83-108.

Dasgupta, Partha y Martin Weale (1992), "On measuring the quality of life”, World Development, 20 (1), Pergamon Press, Great Britain, pp. 119-131.

Desai, Meghnad (1993), "Income and alternative measures of well-being", en David Westendorff y Dharam Ghai (eds.), Monitoring social progress in the 1990s: data constraints, concerns and priorities, UNRISD, Geneva, pp. 23-39.

Despotis, Dimitris (2005), "A reassessment of the Human Development Index via data envelopment analysis", Journal of the Operational Research Society, 56 (8), Operational Research Society, Birmingham, pp. 969-980.

Di Pasquale, Eugenio (2008), "La operacionalización del concepto de Bienestar Social: un análisis comparado de distintas mediciones", 
Observatorio Laboral Revista Venezolana, 1 (2), Universidad de Carabobo, Valencia, Venezuela, pp. 17-42.

Dossel, D. y Rukmani Gounder (1994), "Theory and measurement of living levels: Some empirical results for the Human Development Index", Journal of International Development, 6 (4), Wiley, pp. 415-435.

Doyal, Len y Ian Gough (1991), A theory of human need, Palgrave Macmillan, London UK.

Duarte, Tito y Ramón Elías Jiménez (2007), "Aproximación a la teoría del bienestar", Scientia et Technica, 13 (37), Universidad Tecnológica de Pereira, Pereira, pp. 305-310.

Emrouznejad, Ali, Ibrahim Osman y Abdel Anouze (eds.) (2010), Performance management and measurement with data envelopment analysis, American University of Beirut, Lebanon, <http://www. deazone.com/proceedings/DEA2010-Proceedings.pdf $>, 15 \mathrm{de}$ noviembre de 2015 .

Espejel, Adelina, Iris González y Eva Perón (2004), “El índice de deterioro ambiental en los municipios de Tlaxcala: una propuesta metodológica”, Gaceta ecológica, núm. 70, Secretaría de Medio Ambiente y Recursos Naturales, México, pp. 19-30.

Farrell, Michael J. (1957), "The measurement of productive efficiency", Journal of the Royal Statistical Society, 120 (3), Wiley, pp. 253-290.

Fernández, Tabaré y Emilio Blanco (2004), “¿Cuánto importa la escuela?: el caso de México en el contexto de América Latina”, REICE: Revista Electrónica Iberoamericana sobre Calidad, Eficacia y Cambio en Educación, 2 (1), Red Iberoamericana de Investigación sobre Cambio y Eficacia Escolar, pp. 1-27.

Frey, Bruno y Alois Stutzer (2002), Happiness and economics, Princeton University Press, Princeton.

Giménez, Víctor (2000), "Eficiencia en costes y calidad en la universidad. Una aplicación a los departamentos de la UAB”, tesis doctoral, Universidad Autónoma de Barcelona, Barcelona. 
Goñi, Salomé (1998), "El análisis envolvente de datos como sistema de evaluación de la eficiencia técnica de las organizaciones del sector público: Aplicación en los equipos de atención primaria”, Revista Española de Financiación y Contabilidad, XXVII (97), Asociación Española de Contabilidad y Administración de Empresas, Essex, pp. 979-1004.

Gormley, Patrick (1995), “The Human Development Index in 1994: Impact of income on country rank", Journal of Economic and Social Measurement, 21 (4), IOS Press, Landsdale, pp. 253-267.

Haq, Mahbub ul (1995), Reflections on human development, Oxford University Press, New York.

Hashimoto, Akihiro y Migaku Kodama (1997), "Has livalidity of Japan gotten better for 1956-1990?: a DEA approach", Social Indicators Research, 40 (3), Springer, Berlin, pp. 359-373.

Hashimoto, Akihiro y Hitoshi Ishikawa (1993), "Using DEA to evaluate the state of society as measured by multiple social indicators", Socio-Economic Planning Sciences, 27 (4), Elsevier, pp. 257-268.

Hicks, Douglas (1997), "The inequality-adjusted human development index: a constructive proposal", World Development, 25 (8), Pergamon, pp. 1283-1298.

Inegi (Instituto Nacional de Estadística y Geografía) (2013a), "Censos y conteos de población y vivienda”, Inegi, México, <http://www. inegi.org.mx/est/contenidos/Proyectos/ccpv/default.aspx>, 15 de noviembre de 2015.

Inegi (Instituto Nacional de Estadística y Geografía) (2013b), "Estadística de finanzas públicas estatales y municipales”, Inegi, México, $<$ http://www.inegi.org.mx/sistemas/olap/proyectos/bd/consulta. asp?p=10961\&c=23707\&s=est\&cl=4\#>, 15 de noviembre de 2015 .

Inegi (Instituto Nacional de Estadística y Geografía) (2013c). "Encuesta Nacional de Ocupación y Empleo”, Inegi, México, <http://www. inegi.org.mx/est/contenidos/proyectos/encuestas/hogares/regulares/enoe/default.aspx>, 15 de noviembre de 2015. 
Inegi (Instituto Nacional de Estadística y Geografía) (2013d), "PIB y Cuentas Nacionales", Inegi, México, <http://www.inegi.org.mx/ est/contenidos/proyectos/cn/>, 15 de noviembre de 2015 .

Inegi (Instituto Nacional de Estadística y Geografía) (2013e), "Recursos para la educación”, Inegi, México, <http://www3.Inegi.org.mx/ sistemas/temas/default.aspx?s=est \&c=19004>, 15 de noviembre de 2015.

Jahanshahloo, Gholam, Farhad Hosseinzadeh, Abbas Noora y Bijan Rahmani (2011), "Measuring human development index based on Malmquist productivity index", Applied Mathematical Sciences, 5 (62), Springer, Berlin, pp. 3057-3064.

Jasek-Rysdahl, Kelvin (2001), “Applying Sen's capabilities framework to neighborhoods: using local asset maps to deepen our understanding of well-being", Review of Social Economy, 59 (3), Taylor \& Francis, pp. 313-329.

Lee, Hsuan-Shih, Kuang Lin y Hsin-Hsiung Fang (2006), "A fuzzy multiple objective DEA for the human development index", en B. Gabrys, R. Howlett y L. Jain (eds.), Knowledge-Based Intelligent Information and Engineering Systems, Springer, Berlin, pp. 922-928.

León, Mauricio (2002), "Desarrollo humano y desigualdad en el Ecuador", Gestión, núm. 102, Sistema Integrado de Indicadores Sociales del Ecuador, <http://www.siise.gob.ec/siiseweb/PageWebs/pubsii/ pubsii_0009.pdf>, 15 de noviembre de 2015.

López-Calva, Luis, Lourdes Rodríguez-Chamussy y Miguel Székely (2004), "Medición del Desarrollo Humano en México. Introducción”, Estudios sobre Desarrollo Humano, núm 2003-6, PNUD, México, <http://sic.conaculta.gob.mx/documentos/1006.pdf>, 15 de noviembre de 2015.

López-Vizcaíno, María y Patricio Sánchez-Fernández (2009), "La medición de la calidad de vida en las comarcas gallegas", Revista Galega de Economía, 18 (1), Universidad de Santiago de Compostela, Santiago de Compostela, pp. 1-20.

Mahlberg, Bernhard y Michael Obersteiner (2001), "Remeasuring the HDI by data envelopement analysis", Interim Report, IR-01-069, 
International Institute Applied Systems Analysis, Laxemburg, $<$ http://papers.ssrn.com/sol3/papers.cfm?abstract_id=1999372>, 12 de noviembre de 2015.

Martín, Raquel (2008), "La medición de la eficiencia universitaria: una aplicación del análisis envolvente de datos”, Formación Universitaria, 1 (2), Centro de Información Tecnológica, La Serena, pp. 17-26.

McGillivray, Mark y Howard White (1993), "Measuring development? The UNDP's Human Development Index", Journal of International Development, 5 (2), Wiley, pp. 183-192.

McGillivray, Mark (1991), “The Human Development Index: yet another redundant composite development indicator?", World Development, 19 (10), Pergamon Press, pp. 1461-1468.

Millán, Henio (2001), "Política social y teoría de la justicia. Los fundamentos del combate a la pobreza extrema en México", Economía Sociedad y Territorio, III (9), El Colegio Mexiquense A. C., Zinacantepec, pp. 63-92.

Miranda, Juan Carlos y Lorena del Carmen Araya (2003), "Eficiencia económica en las escuelas del mece/rural desde la perspectiva del análisis envolvente de datos (DEA)", Estudios Pedagógicos, núm. 29, Universidad Austral de Chile, Valdivia, pp. 27-37.

Montenegro, Santiago Javier (2005), "Capacidad fiscal del os gobiernos territoriales colombianos", Colombia, Departamento Nacional de Planeación, República de Colombia, Bogotá, <https://colaboracion.dnp.gov.co/CDT/Desarrollo\%20Territorial/CapacidadFiscal_2004.pdf>, 15 de noviembre de 2015.

Montoya, Omar (2007), "Aplicación del análisis factorial a la investigación de mercados. Caso de estudio", Scientia et Technica, XVIII (35), Universidad Tecnológica de Pereira, Pereira, pp. 281-286.

Murias, Pilar, Fidel Martínez y Simone Novello (2009), "Bienestar económico regional: un enfoque comparativo entre regiones españolas e italianas", Investigaciones Regionales, núm. 18, Asociación Española de Ciencia Regional, Barcelona, pp. 5-36. 
Murray, Christopher (1991), "Development data constraints and the Human Development Index", discussion paper No. 25, UNRISD, Switzerland, <http://www.unrisd.org/80256B3C005BCCF9/ht tpNetITFramePDF?ReadForm\&parentunid=C1CA9D172A23 FB6880256B67005B6239\&parentdoctype=paper\&netitpath $=8$ 0256B3C005BCCF9/(httpAuxPages)/C1CA9D172A23FB688 0256B67005B6239/\$file/dp25.pdf>, 15 de noviembre de 2015.

Narro, José, David Moctezuma y Diego de la Fuente (2013), "Descalabros y desafíos de la política social en México", Problemas del desarrollo, 44 (174), Universidad Nacional Autónoma de México, México, pp. 9-34.

Navarro, José César (2005), Eficiencia del sector eléctrico en México, Instituto de Investigaciones Económicas y Empresariales de la Universidad Michoacana de San Nicolás de Hidalgo, Morelia.

Navarro, José César y Zacarías Torres (2003), "La evaluación de la frontera de eficiencia en el sector eléctrico: un análisis de la frontera de datos (DEA)", Ciencia Nicolaita, núm. 35, Universidad Michoacana de San Nicolás de Hidalgo, Morelia, pp. 39-58.

OCDE (Organización para la Cooperación y el Desarrollo Económico) (2014), Society at a Glance 2014. OECD Social Indicators. The crisis and its aftermath, OECD Publishing, Paris.

Pardo, José Manuel e Isabel García (2004), “Los indicadores de gestión en el ámbito municipal: Implantación, evolución y tendencias", Revista iberoamericana de contabilidad de gestión, núm. 4, Asociación Española de Contabilidad y Administración de Empresas-Asociación Interamericana de Contabilidad, Madrid, pp. 149-180.

Passanante, María Inés (2009), "El desarrollo humano en la Argentina", ponencia presentada en el II Encuentro Nacional de Docentes Universitarios Católicos, Federación Argentina de Universidades Católicas, 26-28 de octubre, Buenos Aires, <www.enduc.org.ar/ comisfin/ponencia/210-03.doc>, 15 de noviembre de 2015.

Pena-Trapero, Bernardo (2009), "La medición del Bienestar Social: una revisión crítica”, Estudios de Economía Aplicada, 27 (2), Asociación Internacional de Economía Aplicada, Valladolid, pp. 299-324. 
Pinzón, Jennifer (2003), "Medición de Eficiencia Técnica Relativa en Hospitales Públicos de Baja Complejidad Mediante la Metodología Data Envelopment Analysis (DEA)”, tesis de magíster, Pontificia Universidad Javeriana, Bogotá, <https://colaboracion. dnp.gov.co/CDT/Estudios\%20Econmicos/245.pdf>, 15 de noviembre de 2015.

PNUD (Programa de las Naciones Unidas para el Desarrollo) (2015), "Sobre el desarrollo humano", PNUD, <http://hdr.undp.org/es/ content/sobre-el-desarrollo-humano>, 15 de noviembre de 2015.

PNUD (Programa de las Naciones Unidas para el Desarrollo) (2013), "Indicadores de desarrollo humano y cuadros temáticos", PNUD, $<$ http://hdr.undp.org/es/data>, 15 de noviembre de 2015.

PNUD (Programa de las Naciones Unidas para el Desarrollo) (2011), "Informe sobre Desarrollo Humano, México 2011", PNUD, <http://hdr.undp.org/sites/default/files/nhdr_mexico_2011.pdf>, 15 de noviembre de 2015.

Prior, Diego y Jordi Surroca (2004), "Eficiencia y sector público: Cómo mejorar el control de la gestión pública", RAE: Revista Asturiana de Economía, núm. 31, Asociación Asturiana de Estudios Económicos, Avilés, pp. 51-68.

Ramos, Xavier y Jacques Silber (2005), "On the application of efficiency analysis to the study of the dimensions of human development", Review of Income and Wealth, 52 (2), Wiley, pp. 285-309.

Ravallion, Martin (2012), “Troubling tradeoffs in the Human Development Index", Journal of Development Economics, 99 (2), Elsevier, B.V. Amsterdam, pp. 201-209.

Reig, Ernest y Ángel Soler (2009), "El índice de desarrollo humano de las regiones españolas: Un enfoque DEA-Multicriterio”, ponencia presentada en la XXV Reunión de Estudios Regionales, 26 y 27 de noviembre, Valencia, España, <http://www.reunionesdeestudiosregionales.org/valencia2009/htdocs/pdf/p238.pdf>, 12 de noviembre de 2015. 
Rojas, Mariano (ed.) (2015), Handbook of Happiness Research in Latin America, International Handbooks of Quality of life-Springer, Dordrecht Heidelberg New York London.

Rojas, Mariano (2010), "Mejorando los programas de combate a la pobreza en México: del ingreso al bienestar", Perfiles latinoamericanos, 18 (35), Flacso, México, pp. 35-59.

Sánchez, María Ángeles y Noelina Rodríguez (2003), “El bienestar social en los municipios andaluces en 1999", RAE: Revista Asturiana de Economía, núm. 27, Asociación Asturiana de Estudios Económicos, Avilés, pp. 99-119.

Scott, John (2011), "Gasto público y desarrollo humano en México: análisis de incidencia y equidad", documento de trabajo para el informe sobre desarrollo humano México, Informe sobre Desarrollo Humano México, 2011, PNUD, México, <http://www. paginaspersonales.unam.mx/files/165/gasto_publico_john_scott. pdf>, 11 de noviembre de 2015.

Secretaría de Salud (2013a), "Gasto Público en Salud 1990-2009", Secretaría de Salud, México, <http://www.sinais.salud.gob.mx/recursosfinancieros/gastopublico.html>, 11 de junio de 2013.

Secretaría de Salud (2013b), "Información dinámica en formato de cubo", Secretaría de Salud, México, <http://www.sinais.salud.gob.mx/ basesdedatos/cubos.html>, 14 de junio de 2013.

SEP (Secretaría de Educación Pública) (2013a), "Estadística e Indicadores Educativos por Entidad Federativa”, SEP, México, <http:// www.snie.sep.gob.mx/indicadores_x_entidad_federativa.html>, 15 de noviembre de 2015 .

SEP (Secretaría de Educación Pública) (2013b), "Información Estadística e Indicadores Educativos", SEP, México, <http://planeacion. sep.gob.mx/estadistica-e-indicadores/estadisticas-e-indicadores>, 15 de noviembre de 2015 .

Sen, Amartya (2014), Collective choice and social welfare, Elsevier, The Netherlands. 
Sen, Amartya (2001), Development as freedom, Oxford University Press, Oxford, 2000, Madrid.

Sen, Amartya (1991), "Welfare, preference and freedom", Journal of Econometrics, 50 (1), Elsevier, pp. 15-29.

Serra, Daniel (2004), Métodos cuantitativos para la toma de decisiones, Ediciones Gestión, Barcelona.

Setien, María Luisa (1989), "La calidad de vida y su medida. Sistema de indicadores sociales para el País Vasco", tesis doctoral, Universidad de Deusto, Bilbao.

Shetty, Udaya y T. Pakkala (2010), "Multistage method of measuring human development through improved directional distance formulation of data envelopment analysis: application to Indian States", Opsearch, 47 (3), Springer, pp.177-194.

Somarriba, Noelia y Bernardo Pena-Trapero (2009), "La medición de la calidad de vida en Europa, el papel de la información subjetiva", Estudios de Económica Aplicada, 27 (2), Asociación Internacional de Economía Aplicada, Valladolid, pp. 373-396.

Srinivasan, Thirukodikaval (1994), "Human Development: A new paradigm or reinvention of the wheel?", The American Economic Review, 84 (2), American Economic Association, Pittsburgh, pp. 238-243.

Streeten, Paul (1994), "Human development: means and ends", The American Economic Review, 84 (2), American Economic Association, Nashville, pp. 232-237.

Trabold-Nubler, Harald (1991), "The Human Development Index-A new development indicator?”, Intereconomics, 26 (5), Springer, pp. 236-243.

Valencia, Enrique (2015), "Los debates sobre los regímenes de bienestar en América Latina y en el Este de Asia. Los casos de México y Corea del Sur", Espiral. Estudios sobre Estado y sociedad, 17 (47), Universidad de Guadalajara, Guadalajara, pp. 65-103.

Veenhoven, Ruut (1998), "Qualita della vita e felicita”, en Girolano de G., A. Becchi, F. Coppa, D. de Leo, G. Neri, P. Rucci y P. Scocco 
(eds.), Salute e qualita della vita, Centro Scientifico Editore, Torino, pp. 67-95.

Wu, Po-Chin, Chiung-Wen Fan y Sheng-Chieh Pan (2014), "Does Human Development Index provide rational development rankings? Evidence from efficiency rankings in super efficiency model", Social Indicators Research, 116 (2), Springer, pp. 647-658.

Yago, Miguel Esteban, Matilde Lafuente y Antonio Losa (2010), "Una aplicación del análisis envolvente de datos a la evaluación del desarrollo. El caso de las entidades federativas de México", en Aceves, Liza, Jaime Estay, Pedro Noguera y Eugenio Sánchez (coords.), Realidades y debates sobre el desarrollo, Universidad de Murcia, Murcia, pp. 119-142.

Zarzosa, Pilar y Noelia Somarriba (2013), "An assessment of social welfare in Spain: territorial analysis using a synthetic welfare indicator”, Social Indicators Research, 111 (1), Springer, pp. 1-23.

Recibido: 22 de agosto de 2014. Corregido: 16 de noviembre de 2015. Aceptado: 10 de febrero 2016.

José César Lenin Navarro-Chávez. Mexicano. Doctor en Ciencias con especialidad en Ciencias Administrativas por la Escuela Superior de Comercio y Administración del Instituto Politécnico Nacional. Posgraduado en Economía y Política Internacional en el Centro de Investigación y Docencia Económicas (CIDE). Actualmente es profesor-investigador del Instituto de Investigaciones Económicas y Empresariales de la Universidad Michoacana de San Nicolás de Hidalgo. Forma parte del Sistema Nacional de Investigadores (SNI) del Consejo Nacional de Ciencia y Tecnología (Conacyt) en México. Sus intereses de investigación se centran en la productividad y competitividad regional y sectorial, así como en la distribución del ingreso y bienestar social. Entre sus últimas publicaciones destacan: Migración, remesas y distribución del ingreso en México y Michoacán, Universidad de Guadalajara-Centro Universitario de Ciencias Económico Administrativas-Universidad Michoacana de San Nicolás de Hidalgo-UCLA Programa on Mexico-PROFMEX/World-Juan Pablos Editor, México (2013); "Public and private investment in the port sector in Mexico, 2000-2010: a study through Data Envelopment Analysis", Chinese Business Review, 13 (1), US-China Business Council, pp. 34-41 
(2014); "Inequality and gender education in America, 1990-2010", European Journal of Social Sciences, 40 (2), Social Sciences Research Platform, Victoria-Mahé, pp. 303-311 (2013); "La desigualdad de la educación en México, 1990-2010: el caso de las entidades federativas", Revista Electrónica de Investigación Educativa, 15 (2), Universidad Autónoma de Baja California, Baja California, pp. 21-33 (2013).

Francisco Javier Ayvar-Campos. Mexicano. Doctor en Ciencias del Desarrollo Regional por la Universidad Michoacana de San Nicolás de Hidalgo. Actualmente es profesor-investigador del Instituto de Investigaciones Económicas y Empresariales de la Universidad Michoacana de San Nicolás de Hidalgo. Forma parte del Sistema Nacional de Investigadores (SNI) del Consejo Nacional de Ciencia y Tecnología (Conacyt) en México. Su línea de investigación actual es desarrollo humano y bienestar social. Entre su últimas publicaciones destacan, en coautoría: "Eficiencia en la generación de educación en Michoacán, 1990-2010: un estudio a través de la envolvente de datos", en A. Sánchez y P. Serrano (coords.), XVIII Encuentro Nacional sobre Desarrollo Regional en México, AMECIDER, México, pp. 1-23 (2013); “Análisis comparativo de los niveles de productividad ente los sectores manufactureros de México, Estados Unidos y Alemania", Revista de Investigaciones México-Estados Unidos (CIMEXUS), VIII (1), Universidad Michoacana de San Nicolás de Hidalgo, Morelia, pp. 13-27 (2013); "Remesas y distribución del ingreso en México y sus regiones, 2000-2010: Un análisis con microdatos”, en C. Tomás y J. Navarro (coords.), Migraciones centroamericanas: Realidades, tendencias $y$ desafios, Universidad Michoacana de San Nicolas de Hidalgo, Morelia, pp. 29-42 (2013).

Víctor Manuel Giménez-García. Español. Doctor en Ciencias Económicas y Empresariales por la Universitat Autònoma de Barcelona. Actualmente es profesor-investigador en el Departamento de Empresa de la Universitat Autònoma de Barcelona, así como consultor independiente de empresas. Su línea de investigación actual es eficiencia, desarrollo humano, simulación y optimización gestión stocks. Entre sus últimas publicaciones destacan: "A comparative analysis of the efficiency of national education systems", Asia Pacific Education Review, núm. 13, Springer, pp.1-15 (2012); "Desempeño de los centros educativos: ¿un problema de recursos o capacidades organizativas?”, Hacienda Pública Españolal Revista de Economía Pública, 198 (3), Instituto de Estudios Fiscales, Madrid, pp. 81-118 (2011); "Optimizing stochastic production-Inventory systems: a heuristic based on simulation and regression analysis", European Journal of Operational Research, núm. 213, Elsevier, pp. 107-118 (2011). 